\title{
Non-alcoholic fatty liver disease (NAFLD) diagnosis and management-differentiating the essential from the ancillary and the present from the future
}

\author{
Amedeo Lonardo ${ }^{1}$, Simonetta Lugari ${ }^{2}$, Fabio Nascimbeni ${ }^{1}$ \\ ${ }^{1}$ Operating Unit Internal Medicine, AOU, Ospedale Civile di Baggiovara, Modena, Italy; ${ }^{2}$ University of Modena and Reggio Emilia, Modena, Italy \\ Correspondence to: Amedeo Lonardo, MD. Simple Operating Unit Metabolic Syndrome, Director, Ospedale Civile, Via Giardini 1135, Modena \\ 41100, Italy. Email: a.lonardo@libero.it. \\ Provenance and Peer Review: This article was commissioned by the editorial office, Hepatobiliary Surgery and Nutrition. The article did not undergo \\ external peer review. \\ Comment on: Barbara M, Scott A, Alkhouri N. New insights into genetic predisposition and novel therapeutic targets for nonalcoholic fatty liver \\ disease. Hepatobiliary Surg Nutr 2018;7:372-81.
}

Submitted Oct 22, 2019. Accepted for publication Nov 05, 2019.

doi: 10.21037/hbsn.2019.11.12

View this article at: http://dx.doi.org/10.21037/hbsn.2019.11.12

"If you do not think about your future, you cannot have one."

_John Galsworthy

\section{Background}

Nonalcoholic fatty liver disease (NAFLD), the spectrum of hepatic and extra-hepatic manifestations and complications resulting from the ectopic deposition of intra-hepatocyte fatty substrates, is a modern epidemic $(1,2)$. NAFLD is not a problem restricted to the USA and/or Europe: compared to the estimated global prevalence of NAFLD, i.e., $25.24 \%$, the Middle East (31.8\%) and South America (30.5\%) display even higher values while Africa ranks significantly below the global threshold (13.5\%) but is not completely exempt from the NAFLD burden (2).

These data clearly document that every practicing physician (not only hepatologists) in every corner of the world (not only in the western hemisphere) must/ should be culturally equipped to promptly identifying those individuals with suspected NAFLD. Physicians must be aware of the pathogenic determinants of disease and contrast them with tailored therapeutic interventions spanning from lifestyle changes, in all patients, to more personalized drug treatment (e.g., antidiabetic, statins, and other agents) in a variable proportion of patients.

\section{The paper by Barbara}

In their recent review article, Doctors Barbara, Scott and Alkhouri (3) first overview the genetic and epigenetic determinants of NAFLD development and progression, the roles of polymorphisms of the four most common genes deemed to be involved in the pathogenesis of NAFLD (PNPLA3; TM6SF2; MBOAT7; and GCKR). Next, they examine the potential to develop such notions into innovative paradigms to be introduced into the arena of diagnosis, prognostication and management of individuals with NAFLD. In this connection, data from pre-clinical studies regarding four promising novel drug agents (Obeticholic acid; Elafibranor; Cenicriviroc; and Selonsertib) and not yet licensed for use in nonalcoholic steatohepatitis (NASH) are also extensively discussed.

\section{Comments}

Let us put ourselves in the shoes of a putative practicing physician who, having read this article, feels heavily discouraged on the grounds that he/she does not have any access to the laboratory testing of gene polymorphisms nor to the enrollment of their NAFLD patients into trials investigating novel drug agents. What could be said to 


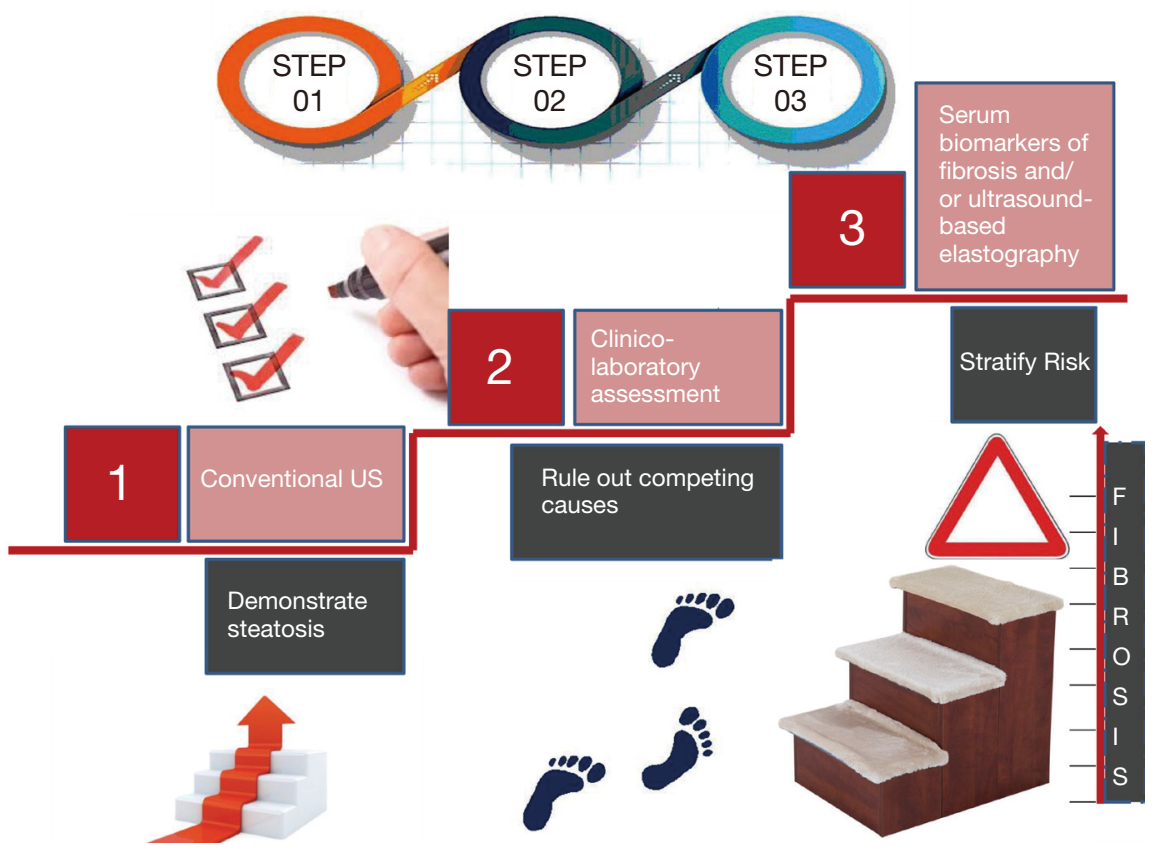

Figure 1 A three-step conceptualization of the diagnostic approach to NAFLD (4,6-10). In at risk individuals, who come to medical observation either through individual consultation or in the setting of screening campaigns, there are three steps to be taken sequentially. Step 1: conventional ultrasonography (US) plays a key role (10). Although, other laboratory tests may also be used to this end (6), US offers the additional advantage of ruling out focal liver disease. Step 2: clinical (e.g., careful collection of historical data through interviews with patients and relatives focusing on occult alcohol consumption) and laboratory assessment (exclusion of infection with hepatitis viruses, autoimmune liver disease, common endocrinopathies, genetic diseases involving the metabolism of heavy metals, rare metabolic disorders). Step 3: various techniques, including chemical and physical may help in identifying those individuals who, based on suspected advanced fibrosis/rapidly fibrosing liver disease need to be submitted to liver biopsy (4). The use of different methods is always guided by clinical judgement and necessarily influenced by the local availability of diagnostic techniques and resources to be allocated to the individual patient in a given clinical or research setting. NAFLD, non-alcoholic fatty liver disease.

encourage this putative physician to continue assisting his/ her NAFLD patients?

\section{Diagnosis of NAFLD (role of genetics)}

NAFLD reflects the paradox of being an extraordinarily widespread disease which, by definition, requires liver biopsy, i.e., an invasive diagnostic procedure (4). Therefore, not surprisingly, clinicians and investigators have identified an array of non-invasive diagnostic techniques which can be classified into either "wet", "biological", serum-based biomarkers or "dry", namely physical techniques, i.e., based on physical characteristics of the liver such as liver stiffness (5). Current guidelines maintain that conventional liver ultrasonography retains a first-line role in discriminating whether steatosis is present or absent $(6,7)$. Further directions as to the suggested staging and follow-up (including the decision to refer the patient for hepatological evaluation and liver biopsy) will ensue as a function of the information obtained by the use of additional non-invasive techniques (i.e., serum biomarkers or ultrasound-based elastography) able to estimate the risk of significant fibrosis (6). To update this step in the correct diagnostic flow-chart, it should be remembered that ultrasound-based elastography, such as vibrationcontrolled transient elastography (VCTE), is reported to have superior diagnostic performance, as compared to serum biomarkers, such as the aspartate aminotransferase-to-platelet ratio index (APRI), NAFLD fibrosis score or the Fib-4, in detecting cirrhosis (8). Guidelines also state that, although genotyping may be considered in selected patients and clinical studies, its use is neither recommended nor advocated routinely $(6,9)$. 


\begin{tabular}{|c|c|}
\hline \multicolumn{2}{|c|}{ APPROVED DRUGS WITH A POTENTIAL ROLE IN NAFLD } \\
\hline Anti-inflammatory/anti-oxidant effect & Vitamin $\mathrm{E}$ \\
\hline Antidiabetic drugs & $\begin{array}{l}\text { Metformin } \\
\text { Pioglitazone } \\
\text { GLP-1 agonists } \\
\text { SGLT-2 inhibitors }\end{array}$ \\
\hline Lipid-lowering drugs & $\begin{array}{l}\text { Statins } \\
\text { Ezetimibe } \\
\text { Fibrates } \\
\text { 3-PUFA }\end{array}$ \\
\hline Antihypertensive drugs & Angiotensin II receptor antagonists \\
\hline \multicolumn{2}{|l|}{ INNOVATIVE AND EXPERIMENTAL DRUGS } \\
\hline \multicolumn{2}{|c|}{ Drugs targeting mainly lipid metabolism and inflammation } \\
\hline FXR agonists & $\begin{array}{l}\text { Obethicolic acid } \\
\text { Tropifexor } \\
\text { Cilofexor }\end{array}$ \\
\hline Dual PPAR $\alpha / \delta$ agonist & Elafibranor \\
\hline CCR2/CCR5 antagonist & Cenicriviroc \\
\hline Acetyl-CoA Carboxylase (ACC) inhibitor & GS-0976 \\
\hline FGF19 analogue & NGM282 \\
\hline Thyroid hormone receptor- $\beta$ agonist & MGL-3196 \\
\hline \multicolumn{2}{|l|}{ Drugs targeting mainly fibrosis } \\
\hline Galectin-3 protein inhibitor & GR-MD-02 \\
\hline Apoptosis signal-regulating kinase 1 (ASK1) inhibitor & Selonsertib \\
\hline Lysyloxidase-like 2 (LOXL-2) monoclonal antibody & Simtuzumab \\
\hline \multicolumn{2}{|l|}{ Other drugs (miscellaneous effects) } \\
\hline $\begin{array}{l}\text { Aramchol } \\
\text { Volixibat } \\
\text { Testosterone undecanoate } \\
\text { Somatropin } \\
\text { Tesamorelin } \\
\text { Metreleptin } \\
\text { Metadoxine } \\
\text { Emricasan } \\
\text { Amlexanox } \\
\text { Tipelukast } \\
\text { Solithromycin } \\
\text { lgG-rich bovine colostrum } \\
\text { Fecal microbiota transplantation }\end{array}$ & \\
\hline
\end{tabular}

Figure 2 Overview of current and future pharmacological therapies for NAFLD/NASH $(1,11)$. NAFLD, nonalcoholic fatty liver disease; NASH, nonalcoholic steatohepatitis.

Figure 1 schematically depicts the conceptual flow to follow in a modern diagnostic approach to NAFLD patients $(4,6-10)$.

\section{Management of NAFLD}

An in-depth understanding of the complex pathogenesis of NAFLD and NASH is preliminary to any rational therapeutic approach (1). As shown in Figure $2(1,11)$, there are several marketed drugs which, although not specifically licensed for use in NASH, show promise in potentially affecting the development of fibrosis, which is the major driver of both the hepatic and extra-hepatic course of NAFLD.

\section{Role of pharmacogenomics in the personalized management of NAFLD}

A recent experimental study showed that an antisense oligonucleotide therapy silencing PNPLA3 was able to improve all the histological features of NAFLD, including inflammation and fibrosis, in a PNPLA3 knock-in 148M/M mutant mice, paving the way to a precision medicine approach based on the suppression of a strong innate 
genetic risk factor (12). Lorbek et al., in their review of the literature, highlight that although this must be confirmed, the V433M variant of CYP4F2 may affect the outcome of treatment with vitamin $\mathrm{E}$; moreover polymorphism I148M of PNPLA3 may affect the outcome of diet change, PUFA treatment and drug therapy in NAFLD patients (13). Along the same line, Kawaguchi-Suzuki et al. have found that a genetic response score based on five SNPs significantly predicted the histological resolution of NASH following pioglitazone administration (14). These examples illustrate the potential for pharmacogenomic research in NAFLD which is in its early days and therefore not yet applicable to clinical practice.

\section{Conclusion and research agenda}

In conclusion, the paper by Doctor Barbara et al. (3) allows us to look at the future of NAFLD diagnosis and management with the use of genotyping and innovative drugs. Meanwhile, the state-of-the-art status of diagnosis of NAFLD should not fail to consider the importance of in-depth clinical assessment based on a priori probability of disease, conventional ultrasonography (10), serum biomarkers and innovative techniques (such as VCTE), if the latter are available, the exclusion of competing causes of liver disease and liver biopsy whenever clinically indicated (4). The mainstay of NAFLD treatment is based on lifestyle changes including dieting and physical activity (6). Drugs licensed as anti-diabetic agents and statins may also play a prominent role given that the vast majority of NASH patients do have pre-diabetes/ diabetes and/or atherogenic dyslipidemia. Finally, the severely obese individual with NASH may safely be offered metabolic/bariatric surgery.

Clearly, in the future, a more tailored approach will be adopted to identify those patients who are genetically more exposed to the risk of NASH and to select the most fruitful drug treatment for each individual patient. Meanwhile, we have to remember that a strong genetic determinant of the natural course of NAFLD is promptly identifiable: sex! (15). Finally, it is our responsibility to provide the best medical assistance to patients based on current standards and to differentiate the essential from the ancillary while keeping in mind that what seems ancillary now may become essential in the future.

\section{Acknowledgments}

Funding: None.

\section{Footnote}

Conflicts of Interest: All authors have completed the ICMJE uniform disclosure form (available at http://dx.doi. org/10.21037/hbsn.2019.11.12). The authors have no conflicts of interest to declare.

Ethical Statement: The authors are accountable for all aspects of the work in ensuring that questions related to the accuracy or integrity of any part of the work are appropriately investigated and resolved.

Open Access Statement: This is an Open Access article distributed in accordance with the Creative Commons Attribution-NonCommercial-NoDerivs 4.0 International License (CC BY-NC-ND 4.0), which permits the noncommercial replication and distribution of the article with the strict proviso that no changes or edits are made and the original work is properly cited (including links to both the formal publication through the relevant DOI and the license). See: https://creativecommons.org/licenses/by-nc$\mathrm{nd} / 4.0 /$.

\section{References}

1. Italian Association for the Study of the Liver (AISF). AISF position paper on nonalcoholic fatty liver disease (NAFLD): Updates and future directions. Dig Liver Dis 2017;49:471-83.

2. Younossi ZM, Koenig AB, Abdelatif D, et al. Global epidemiology of nonalcoholic fatty liver disease-Metaanalytic assessment of prevalence, incidence, and outcomes. Hepatology 2016;64:73-84.

3. Barbara M, Scott A, Alkhouri N. New insights into genetic predisposition and novel therapeutic targets for nonalcoholic fatty liver disease. Hepatobiliary Surg Nutr 2018;7:372-81.

4. Nascimbeni F, Ballestri S, Machado MV, et al. Clinical relevance of liver histopathology and different histological classifications of NASH in adults. Expert Rev Gastroenterol Hepatol 2018;12:351-67.

5. Castera L, Friedrich-Rust M, Loomba R. Noninvasive Assessment of Liver Disease in Patients With Nonalcoholic Fatty Liver Disease. Gastroenterology 2019;156:1264-81.e4.

6. European Association for the Study of the Liver (EASL); European Association for the Study of Diabetes (EASD); European Association for the Study of Obesity (EASO). 
EASL-EASD-EASO Clinical Practice Guidelines for the management of non-alcoholic fatty liver disease. J Hepatol 2016;64:1388-402.

7. Wong VW, Chan WK, Chitturi S, et al. Asia-Pacific Working Party on Non-alcoholic Fatty Liver Disease guidelines 2017-Part 1: Definition, risk factors and assessment. J Gastroenterol Hepatol 2018;33:70-85.

8. Singh S, Muir AJ, Dieterich DT, et al. American Gastroenterological Association Institute Technical Review on the Role of Elastography in Chronic Liver Diseases. Gastroenterology 2017;152:1544-77.

9. Chalasani N, Younossi Z, Lavine JE, et al. The diagnosis and management of nonalcoholic fatty liver disease: Practice guidance from the American Association for the Study of Liver Diseases. Hepatology 2018;67:328-57.

10. Ballestri F, Nascimbeni S, Lugari A, et al. critical appraisal of the use of ultrasound in hepatic steatosis Expert review of gastroenterology \& hepatology 13, 667-81.

Cite this article as: Lonardo A, Lugari S, Nascimbeni F. Non-alcoholic fatty liver disease (NAFLD) diagnosis and management-differentiating the essential from the ancillary and the present from the future. HepatoBiliary Surg Nutr 2020;9(3):374-378. doi: 10.21037/hbsn.2019.11.12
11. Sumida Y, Yoneda M. Current and future pharmacological therapies for NAFLD/NASH. J Gastroenterol 2018;53:362-76.

12. Lindén D, Ahnmark A, Pingitore P, et al. Pnpla3 silencing with antisense oligonucleotides ameliorates nonalcoholic steatohepatitis and fibrosis in Pnpla3 I148M knock-in mice. Mol Metab 2019;22:49-61.

13. Lorbek G, Urlep $\check{Z}$, Rozman D. Pharmacogenomic and personalized approaches to tackle nonalcoholic fatty liver disease. Pharmacogenomics 2016;17:1273-88.

14. Kawaguchi-Suzuki M, Cusi K, Bril F, et al. A Genetic Score Associates With Pioglitazone Response in Patients With Non-alcoholic Steatohepatitis. Front Pharmacol 2018;9:752.

15. Lonardo A, Nascimbeni F, Ballestri S, et al. Sex Differences in Nonalcoholic Fatty Liver Disease: State of the Art and Identification of Research Gaps. Hepatology 2019;70:1457-69. 